\title{
Améliorer la motivation dans les Environnements d'Apprentissage basés sur le jeu
}

\author{
Thibault Carron, Jean-Charles Marty \\ Laboratoire SysCom - Université de Savoie \\ Campus Scientifique, Bâtiment Mont-Blanc, \\ F-73376 Le Bourget du lac cedex \\ thibault.carron@univ-savoie.fr,jean-charles.marty@univ-savoie.fr
}

\begin{abstract}
RÉSUMÉ. Dans cet article, les auteurs se sont intéressés principalement à deux éléments contribuant à la motivation dans les Environnements d'Apprentissage basés sur le jeu: la flexibilité du système et l'immersion des utilisateurs dans le système. Cet article est divisé en trois sections. Le premier concerne les besoins d'adaptation à partir des points de vue à la fois de l'enseignant et de l'apprenant. Les auteurs ont besoin de récolter des traces au sujet des activités pédagogiques afin de proposer des fonctionnalités d'observation pour mettre à jour un modèle usager adapté pour les Learning Games. Ce modèle usager est vu comme un objet collaboratif explicite affiché directement dans le jeu. La seconde section s'intéresse à la nécessité de maintenir les usagers immergés dans le jeu et suggère des lignes directrices pour l'immersion concernant le game design, le gameplay et l'aide par métaphores. La dernière partie illustre ces points à travers un environnement d'apprentissage de type Learning Game appelé "Learning Adventure". La mise en cuvre d'une session d'apprentissage dans cet environnement est décrite ainsi qu'une expérimentation en situation écologique servant de support à l'explication des concepts présentés au-dessus.
\end{abstract}

ABSTRACT. In this article, the authors propose to address two main items contributing to motivation in Game-Based Learning Environments: the flexibility of the system and the immersion of the users in the system. This article is split into three sections. The first one deals with the need for adaptation from both the teacher's and the learners' point of view. The authors need to collect traces about pedagogical activities in order to propose observation features for updating a user model adapted for learning games. This user model is seen as an explicit collaborative object displayed in the game. The second section concerns the necessity of keeping the users immersed in the game and gives some guidelines for immersion concerning game design, game play and metaphorical support. The last part illustrates these points through a game-based learning environment called "learning

\footnotetext{
${ }^{1} \mathrm{http}: / /$ www.learningadventure.eu
}

Revue. Volume $\mathrm{X}-\mathrm{n}^{\circ} \mathrm{x} /$ année, pages 1 à $\mathrm{X}$ 
2 Revue. Volume $\mathrm{X}-\mathrm{n}^{\circ} \mathrm{x} / \mathrm{année}$

adventure". The generation of a learning session in the environment is described and a real experiment is used as a support for explaining the concepts presented above.

MOTS-CLÉS: Learning games, immersion, flexibilité, motivation, adaptation, observation, modèle usager.

KEYWORDS: Learning games, immersion, flexibility, motivation, adaptation, observation, user model. 


\section{Introduction}

De nos jours, comparé aux méthodes d'enseignement traditionnelles, les LMS (Learning Management Systems) offrent désormais des fonctionnalités reconnues selon différents points de vue. Par exemple, les étudiants peuvent apprendre à leur propre rythme. Ces environnements permettent également à l'enseignant d'évaluer des activités spécifiques de manière uniforme. Cependant, bien qu'ils offrent de puissantes fonctionnalités, ils reçoivent également des critiques majeures (manque d'awareness, peu de possibilités en terme de collaboration ou de régulation (KianSam et al., 2002)). De plus, les étudiants ont maintenant tendance à les trouver peu attrayants (Prensky, 2000).

$\mathrm{Au}$ sujet de ce point particulier, en accord avec Vygotski et la théorie de l'activité (Vygotski, 1934), nous considérons que la dimension sociale est cruciale dans le processus cognitif lié à l'activité d'apprentissage. Par conséquent, nous avons cherché à améliorer la prise en compte de la dimension sociale dans ces environnements. L'émergence des Learning Games a offert un moyen de répondre à ce problème et ceux-ci peuvent être vus comme une évolution des «classiques » LMS (Hijon et al., 2006).

Les Learning Games sont déjà recommandés pour un certain nombre de qualités pédagogiques qu'ils possèdent intrinsèquement (Squire, 2003). Par exemple, ils offrent une courbe spécifique d'apprentissage progressive adaptée à chaque apprenant; ils permettent l'apprentissage à distance et peuvent encourager des pratiques spécifiques dans le domaine de l'éducation telles que l'apprentissage collaboratif ou la pédagogie par projet.

De manière plus générale, les jeux vidéo sont même vus comme une nouvelle forme de rhétorique grâce à leur basique "mode représentatif de la procéduralité » (Bogost, 2007) et peuvent être un moyen d'envisager l'apprentissage de la programmation sous un œil nouveau (Matheas, 2005).

Observant l'émergence et constatant le succès des jeux en ligne multi-joueurs auprès des étudiants, nous avons décidé d'expérimenter l'approche d'apprentissage via le jeu, en développant un nouvel environnement de jeu et en l'utilisant comme support pour des sessions d'apprentissage.

Nous nous appuyons sur la métaphore de l'exploration d'un monde virtuel appelé "Learning Adventure" dans lequel chaque étudiant embarque pour une quête afin d'acquérir des connaissances relatives à l'activité d'apprentissage. Nous pensons que la manière d'apprendre dans une session d'apprentissage est similaire au suivi d'une aventure dans un jeu vidéo de rôle (Role-Playing Game ou RPG). La combinaison des deux styles est appelée $\mathrm{MMORPG}^{2}$ et présente un potentiel important pour l'apprentissage (Galarneau et al., 2007) ( $\mathrm{Yu}, 2009)$ que l'on retrouve parfois dans la littérature reformulée sous le terme MMOLE. A ce sujet,

${ }^{2}$ mmorpg : massively multiplayer online role playing game 
nous nous positionnons dans le même esprit que (Buckingham et al., 2007) en considérant l'affordance entre jeu et pratique éducative en essayant de tenir compte à l'ensemble des aspects du jeu qui nécessitent d'être étudiés avec précaution.

Un des facteurs majeurs pour obtenir de bons résultats dans l'activité d'apprentissage réside dans la motivation des apprenants comme on le retrouve dans (Amory et al., 1999)(Kirriemuir et al., 2004)(Galarneau et al., 2007)(Dondlinger, 2007). Dans cet article, nous souhaitons nous focaliser sur deux points principaux qui contribuent à la motivation: la flexibilité du système et l'immersion des utilisateurs dans l'environnement, qui est aussi généralement vu comme un composant fondamental du "'gameplay" (Ermi et al., 2005).

\section{Flexibilité}

Des besoins en adaptation existent aussi bien du point de vue de l'enseignant (adaptation de la difficulté des exercices, ajouts de nouvelles activités, diviser le groupe en sous-groupes) que du point de vue de l'apprenant (la motivation est liée à la nouveauté, la surprise au sens non-répétition - l'enchaînement dans le jeu ne doit pas être le même lorsque l'on démarre l'aventure ou que l'on déjà passé certaines étapes lors de sessions précédentes).

Ces besoins d'adaptation peuvent aussi être catégorisés en deux groupes : ceux qui sont liés à l'adaptation pendant l'activité (besoins en adaptation dynamique) et ceux qui imposent une adaptation après l'activité, telle que l'adaptation nécessaire du jeu pour la session suivante ou des éléments liés au processus métacognitif (besoins en adaptation statique).

La flexibilité consiste ainsi à adapter l'environnement en fonction d'un contexte particulier. Un élément clé dans la description du contexte réside dans l'état courant des apprenants, et nous estimons que la flexibilité repose beaucoup sur l'utilisation d'un modèle usager (user model).

\section{Immersion}

Nous nous focalisons également sur le concept d'immersion car il est fondamental de maintenir une cohérence entre le contenu pédagogique et le jeu lui-même.

En effet, même dans les Learning games les plus récents, notre expérience montre qu'un point particulier n'est quasiment jamais pris en compte : l'ensemble du design du jeu n'est pas lié aux concepts d'apprentissage. C'est la raison pour laquelle les apprenants/joueurs ne se sentent pas immergés dans un monde cohérent pour eux.

Dans cet article, nous commençons par nous intéresser à ces aspects centraux pour la motivation : Flexibilité et Immersion. Puis nous décrirons leur application dans "Learning Adventure", notre environnement d'apprentissage basé sur le jeu. 


\section{Flexibilité et Modèle Usager}

Dans les LMS, le concept de profil d'apprenant ou modèle d'apprenant est utilisé pour représenter les caractéristiques d'un étudiant (Brusilovsky, 2001; Rueda et al., 2003; Vassileva et al., 2003). Naturellement, il est tout aussi important dans un Learning Game (LG) avec une approche RPG dans lequel l'apprenant est représenté directement dans le jeu au moyen d'un avatar.

En effet, dans les RPG, un tel avatar représentant le joueur embarque des caractéristiques individuelles; dans un LG, la représentation de l'apprenant devrait également incorporer des informations pédagogiques dans un objet accessible à la fois pour l'étudiant et l' (les) enseignant(s).

En outre, il devrait être possible d'utiliser dans d'autres environnements d'apprentissage cette information concernant à la fois la connaissance acquise par l'apprenant mais aussi les aspects comportementaux (bavard, coopératif, lent) et même des caractéristiques civiques comme on le trouve dans (Kahne et al., 2009) ou (Salen, 2007) via le RPG «Gamestar Mechanic ». Nous considérons ainsi, que toutes ces informations devraient être rassemblées dans un unique objet (le modèle usager) persistant. Sur ce point, nous sommes d'accord avec l'idée de Vassileva : le modèle usager est décentralisé dans les nouveaux environnements d'apprentissage et appartiennent à l'utilisateur (Vassileva et al., 2003). Le modèle usager peut présenter un moyen de représenter l'apprenant, rassemblant les caractéristiques pertinentes pour l'apprentissage (compétences, comportements et préférences). Cette approche conduit à des systèmes dans lesquels le modèle usager n'est pas directement intégré dans les outils d'apprentissage, mais accessible à travers la communication inter-processus. A ce propos, Kobsa démontre dans (Kobsa, 2001) les avantages d'une telle approche distribuée.

Ces nombreuses caractéristiques peuvent être classées en plusieurs catégories: caractéristiques communes, préférences, compétences, comportements, ou même succès spéciaux («achievements» dans les jeux récents). Nous définissons ainsi dans une première partie ce qui se retrouve à l'intérieur du modèle usager et proposons une classification possible des attributs (caractéristiques) d'un utilisateur. En effet, de précédents travaux, des expérimentations et les retours d'enseignants ont permis d'éliciter plusieurs besoins relatifs à la représentation d'un étudiant dans les learning games. Nous soulignons aussi quelles améliorations du modèle usager actuel nécessitent une attention particulière. Ensuite, nous proposons une représentation graphique $\mathrm{du}$ modèle usager prenant en compte le besoin de flexibilité de tels jeux.

\subsection{Décrire un modèle usager adapté aux $L G$}

Avant tout, un étudiant est identifié et représenté par des informations spécifiques, généralement statiques, particulièrement dans le domaine académique. Ces caractéristiques communes du modèle usager sont importantes d'un point de 
vue administratif et sont constamment présentes dans un modèle usager afin de pouvoir identifier une personne individuellement.

Un étudiant est décrit par son nom, sa carte d'étudiant, des cours actuels ou passés, des diplômes, etc. De telles informations peuvent être rassemblées automatiquement à partir des services administratifs, ou le système peut explicitement les demander à l'utilisateur lorsqu'un nouveau compte est créé dans le LG.

Ces caractéristiques sont souvent utilisées pour adapter une application, rendre le système convivial (l'utilisation du nom, de l'adresse pour trouver des références locales, etc.).

La deuxième catégorie des caractéristiques Utilisateur concerne les préférences de l'utilisateur. Dans cette catégorie, nous pouvons trouver les Préférences d'Interface utilisateur (des polices de caractères, des couleurs) ou les sujets auquel l'utilisateur s'intéresse (s'ils sont en rapport avec l'application, bien sûr).

Certaines préférences peuvent être mises en œuvre pour fournir à l'étudiant des interfaces graphiques adaptées ou une modalité adaptée de présentation de l'information (le texte, l'image, le son, la vidéo) (Brusilovsky, 2001). C'est particulièrement pertinent dans des systèmes pédagogiques où l'acquisition de connaissance est améliorée grâce à un choix d'enseignement adapté aux capacités personnels de mémorisation de l'information (Choukroun et al., 1985) de l'utilisateur (visuel, auditif, format de données, etc.). Cette approche est cependant rarement utilisée parce que le professeur doit fournir les mêmes informations dans beaucoup de formats ou différentes modalités ; tout cela se révèle très coûteux en temps.

La partie du modèle usager contenant les informations sur les compétences de l'utilisateur se réfère à la connaissance acquise par un étudiant. Les caractéristiques contenues dans cette partie changent plus souvent que ceux décrits dans les deux parties précédentes. Les compétences d'utilisateur sont largement utilisées pour personnaliser le chemin conduisant l'acquisition de connaissance et adapter la forme de présentation. (Brusilovsky, 2001) explique en détail comment des relations peuvent être prolongées ou cachées en fonction de la connaissance de l'utilisateur au sujet d'un concept particulier. Dans des jeux vidéo, et particulièrement dans les RPG, la progression dans le jeu est directement contrôlée par les compétences de l'avatar. Par exemple, il est impossible d'atteindre une île si votre avatar ne sait pas nager ou n'a pas d'accès à un bateau ... "La compétence natation" doit être acquise pour explorer cette île. 
Pour rendre nos LG adaptables et permettre les activités requises, nous définissons un modèle usager spécifiquement adapté aux LG sur le même concept : un apprenant est défini avec des compétences (et des niveaux différents pour chaque compétence) qui permettent l'accès à de nouveaux exercices ou à une nouvelle partie $\mathrm{du}$ monde du jeu. Comme dans l'enseignement traditionnel, où quelques exercices exigent des connaissances spécifiques (prérequis), la planification des exercices dépend des compétences de l'étudiant et le monde du jeu est automatiquement adapté à chaque étudiant en fonction du contenu de son modèle usager. Il est possible de voir un niveau de compétence comme une clé permettant l'accès aux exercices suivants (et la partie du monde correspondante).

Finalement, la quatrième partie contient des caractéristiques déduites du comportement d'un utilisateur. Cette partie est particulièrement utile dans des nouveaux systèmes d'étude où la collaboration et les aspects sociaux sont centraux. Il est par exemple, important pour un professeur d'être conscient des comportements différents de ses étudiants : collaboratif, bavard, hésitant, etc.

Ces caractéristiques sont cruciales pour réglementer une activité d'apprentissage (Marty et al., 2007). Par exemple, un professeur peut définir dans son scénario pédagogique (la définition du séquencement des actions dans le jeu) qu'une activité est disponible seulement si la coopération à l'intérieur d'un groupe d'étudiants atteint un certain seuil.

Comme nous pouvons voir, les caractéristiques de l'utilisateur sont nombreuses et c'est un défi pour le concepteur de jeu de les présenter aux utilisateurs sans les obliger à changer leur centre d'attention. En plus des tendances actuelles dans le domaine du modèle usager, il y a un besoin réel de considérer le modèle usager comme un véritable objet, central dans le système. Cet objet est utilisé pour adapter non seulement les aspects d'apprentissage dans le jeu mais aussi le jeu lui-même. Il devra alors être facilement mis à jour en fonction de nouvelles connaissances ou nouvelles compétences de l'utilisateur. Comme un véritable objet réel, nous devrions pouvoir aussi exécuter des actions sur lui, comme des actions d'interaction ou de visualisation.

L'interaction avec le modèle usager ouvre de nouvelles perspectives dans lesquelles l'utilisateur peut agir sur sa propre représentation, exprimant des vœux sur les compétences qu'il souhaiterait améliorer. La visualisation est directement liée avec la représentation visuelle du modèle usager : une représentation adaptée et conviviale du modèle usager est importante pour garder l'apprenant immergé dans le 
jeu. Nous proposons ainsi de lier la formalisation de modèle usager standard (texte, $\mathrm{xml}$ ) avec une représentation graphique de celui-ci. Dans la section suivante, nous expliquons comment les différentes caractéristiques peuvent être représentées graphiquement selon les catégories (définies ci-dessus) et peuvent être utilisées pour adapter l'environnement (le jeu) au contexte.

\subsection{Représenter le modèle usager}

Dans les jeux vidéo classiques et récents comme les RPG (par exemple : World of Warcraft, Dofus), un certain nombre d'informations, de caractéristiques ou de propriétés apparaissent directement dans le jeu affichées sur la représentation de l'avatar. Pour rester immergé dans le jeu, il est crucial que le modèle usager ait une forme (une apparence) liée au jeu. La figure 1 présente un exemple où le modèle usager est représenté sur un parchemin3. Cette approche est utile quand l'utilisateur doit consulter des détails du modèle d'usager (comme quand quelqu'un consulte une carte). Cependant comme nous l'avons précisé, il peut être également approprié de représenter certaines caractéristiques principales directement sur l'avatar de l'apprenant.

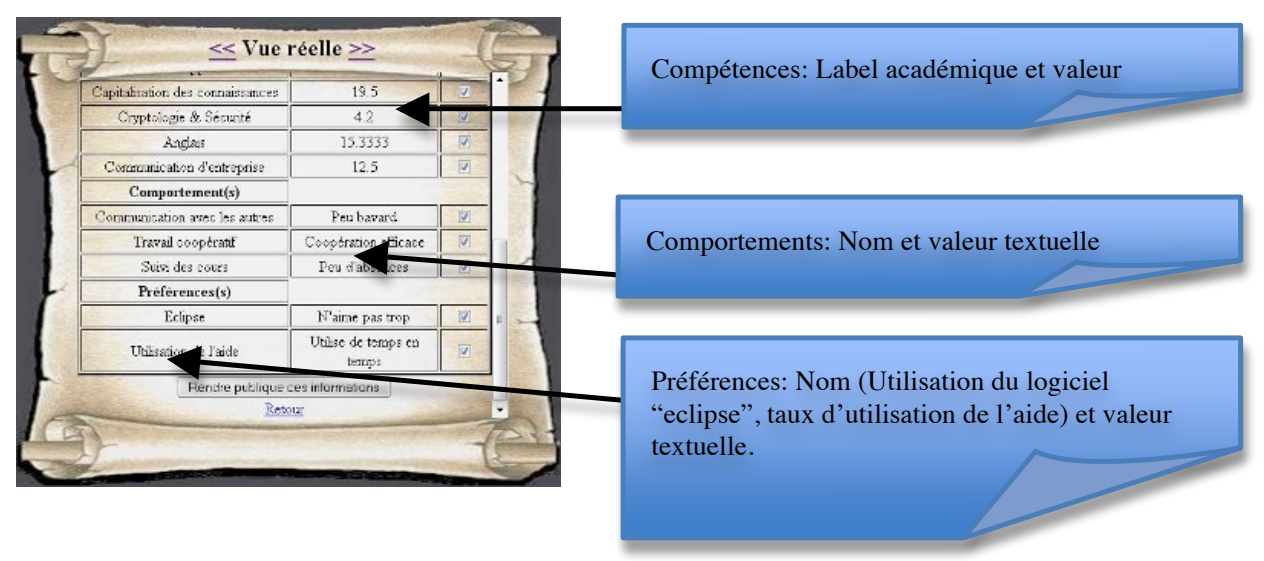

Figure 1. Exemple de modèle usager adapté aux Learning Games.

Voici un exemple dans lequel nous avons représenté quelques caractéristiques des étudiants dans le dernier LG (Carron et al., 2008) que nous avons développés.

\footnotetext{
${ }^{3}$ Pour illustrer notre propos, nous présentons des images d'un modèle usager issues d'un autre learning game que nous avons développé appelé « donjon pédagogique », dans lequel un style « héroïc fantasy » est utilisé.
} 
Dans ce LG, l'enseignant s'est focalisé principalement sur la connaissance acquise par l'étudiant et son comportement. Nous avons ainsi défini une correspondance graphique pour les caractéristiques contenues dans la partie "compétence" et dans la partie "comportement". Quelques éléments (l'épée, le bouclier, le casque, etc.) gagnés ou affichés explicitement sur l'avatar représentent des compétences; nous y avons ajouté une représentation graphique du modèle d'usager pour l'intégration dans le jeu. Un certain nombre d'éléments est localisé sur la représentation de l'avatar pour afficher certaines compétences choisies et spécifiques (dans l'image 2, nous avons six emplacements d'éléments représentées par des points d'interrogation). Comme nous verrons dans la deuxième partie de cet article, pour des questions d'aspect immersif et de motivation, de personnes réelles (connues par les joueurs) sont représentées directement dans le jeu.

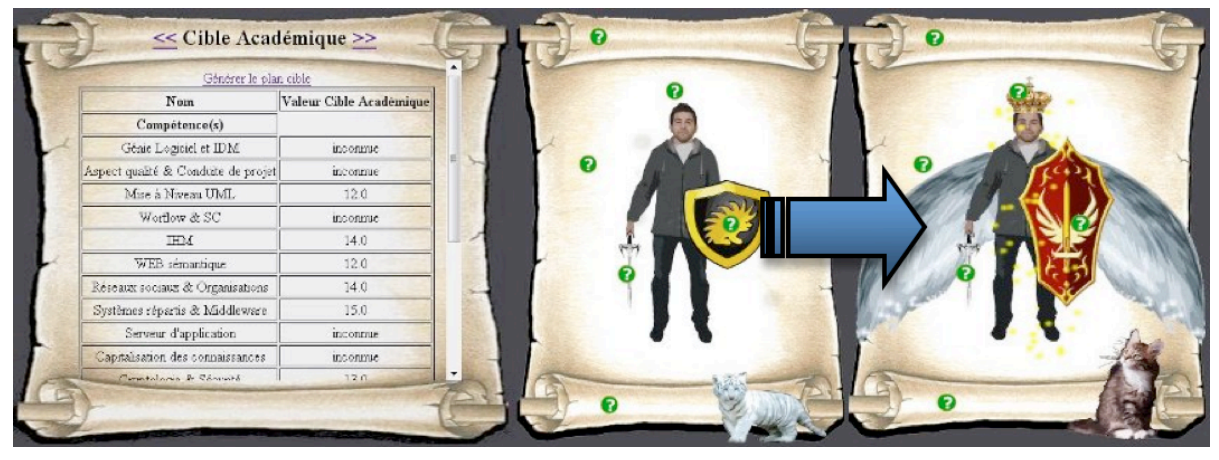

Figure 2. Exemple de représentation graphique

Nous pouvons voir sur la figure 2 qu'il y a une correspondance entre certaines caractéristiques du modèle usager textuel (pour être consulté quand des informations complètes sont nécessaires) et celui de la vue graphique correspondant à l'avatar affiché dans le jeu. Dans la vue complètement à droite de l'image, le même avatar est représenté avec des valeurs plus impressionnantes. Chaque élément peut être représenté avec des affichages différents (plus ou moins impressionnants) pour distinguer différents niveaux concernant une compétence donnée. 
Dans certains cas, il peut être intéressant de représenter graphiquement les comportements aussi. Comme pour nos indicateurs collaboratifs (Gendron, 2008), nous avons voulu représenter des comportements avec des animations différentes autour de l'avatar. La forme, la vitesse, le nombre, la densité, la hauteur et la taille d'éléments animés (voir l'image 3) gravitant autour de la représentation de l'avatar peuvent être utilisés pour donner des informations concernant les comportements mis en évidence par le joueur pour être représentés directement dans le jeu (par exemple les boules jaunes autour de l'avatar dans le fond de l'image 3).

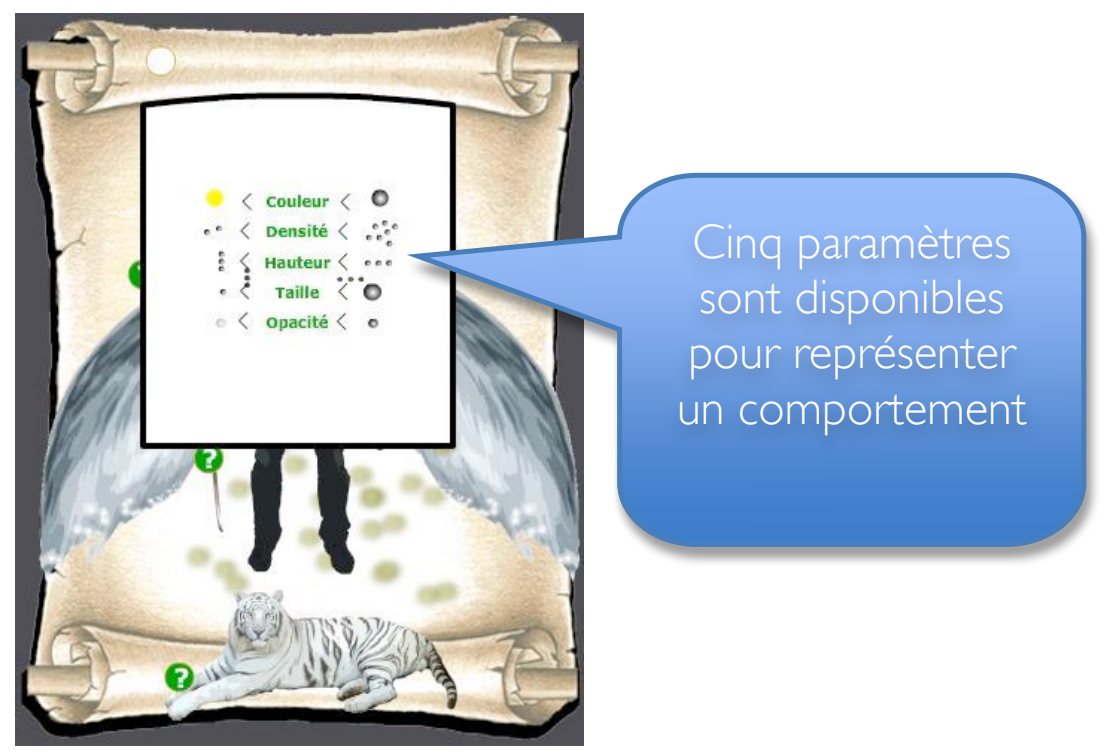

Figure 3. Configurer les éléments animés représentant des comportements spécifiques

Actuellement, la sémantique associée à ces indicateurs visuels (concernant des compétences et des comportements est libre, choisie (et connue) par l'administrateur $\mathrm{du}$ jeu et peut exiger de la pratique pour être naturellement ou intuitivement interprétée dans le jeu.

La visualisation du modèle d'usager est très utile et séduisante pour les utilisateurs. C'est encore plus sensible quand les données sont mises à jour dynamiquement. Dans la section suivante nous présentons comment un tel modèle 
d'apprenant est mis à jour grâce aux traces d'activité laissées par les utilisateurs en utilisant le jeu.

\subsection{Evolution du modèle usager}

Dans des sites web commerciaux ou dans des plates-formes collaboratives pour la gestion des connaissances par exemple, le problème principal et bien connu lié au modèle usager est de le garder mis à jour (Fink et al., 2000). Nous verrons dans cette section comment prendre en compte une telle évolution dans LG.

De notre point de vue, l'observation des traces et les RPG fournissent de nouvelles possibilités de définir un modèle d'apprenant. Notre approche consiste à profiter des traces laissées par les joueurs participant à l'activité d'apprentissage médiée par le jeu pour calculer des indicateurs d'awareness pour le LG (Gendron et al., 2008). En fait, il est ainsi possible d'identifier implicitement la progression dans les compétences, mais aussi des comportements et les préférences des utilisateurs (Carron et al., 2008) et même des comportements interactifs entre les apprenants (Dimitracopoulou et al., 2005; Dimitriadis et al., 2006). Il est par conséquent réalisable de définir et mettre à jour un modèle usager spécifique. Dans un LG et dans notre contexte de travail, le problème est plus facile à résoudre parce que le modèle d'usager est naturellement centré sur les activités d'un apprenant. Une réponse exacte à une activité pédagogique, à un exercice dans le jeu ou même la terminaison d'un scénario dans le LG va apporter des points et modifiera la valeur de la compétence correspondante dans le modèle d'apprenant.

Comme indiqué dans (Carron et al., 2008), il est possible d'obtenir des informations à partir des traces laissées par les utilisateurs en utilisant le jeu. L'idée de base de notre approche est d'avoir des sondes fournissant des informations sur des événements de base arrivant dans le LG (par exemple l'entrée dans une pièce, la discussion avec quelqu'un) et de les composer pour avoir des affirmations plus générales (par exemple « cet étudiant répond correctement, mais travaille seul). La définition de règles spécifiques laissera le système utiliser une trace particulière pour mettre à jour une (ou plusieurs) caractéristiques spécifiques du modèle usager dans le jeu (cf. figure 4). 


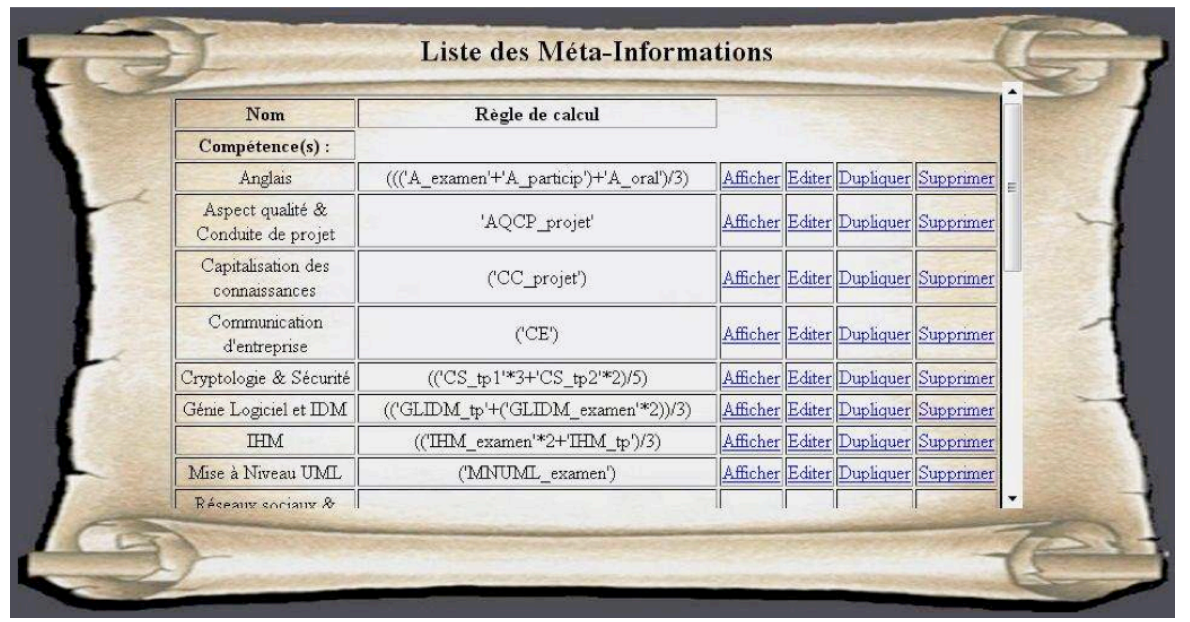

Figure 4. Liste des règles relatives à la mise à jour des compétences

Nous équipons généralement nos LG d'une possibilité de traçage basée sur une architecture d'observation multi-agents (cf. figure 5) (Carron et al., 2006; Loghin et al., 2008). Les actions comme répondre correctement à un QCM ou utiliser l'outil de messagerie instantanée peuvent être tracées et ainsi récupérées au moyen de sondes élémentaires. Chaque sonde contient des paramètres et, en fonction de ceux-ci, un but particulier afin d'améliorer une ou plusieurs caractéristiques apparaissant dans le modèle usager. Par exemple, lorsqu'un seuil concernant le comportement bavard (n messages envoyés) est atteint, la partie locale du modèle d'usager est mise à jour grâce aux règles ${ }^{4}$ (voir 1 dans la figure 5), puis propagée à la version complète sur un serveur (voir 2 dans la figure 5) et aux clients concernés (par exemple l'enseignant), éventuellement directement à l'intérieur du jeu via des indicateurs (voir 3 dans la figure 5).

\footnotetext{
${ }^{4}$ Par exemple, Règle ::'NombreDeMessages $>20$ ’ => comportement(bavard, haut)
} 


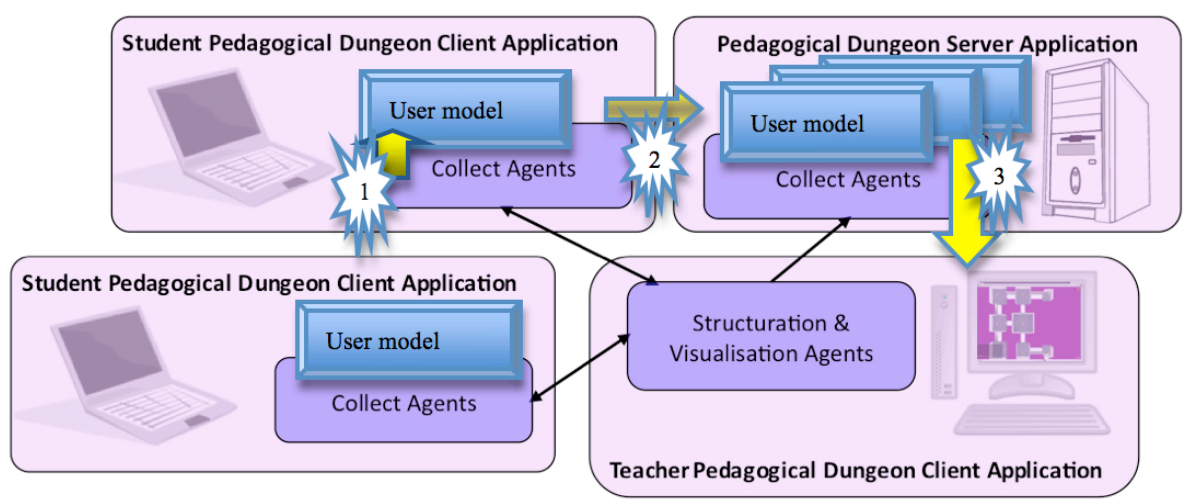

Figure 5. Architecture d'observation multi-agents

Bien que l'on considère généralement le modèle usager comme une vue privée, plus ou moins réaliste d'un utilisateur, nous proposons de tirer partie de l'observation dans les LG et d'étendre notre vision du modèle usager adapté à un jeu LG en ajoutant un autre point fondamental spécifique à notre modèle d'apprenant. Il concerne le concept de vues futures interactives. Comme nous le verrons, les règles peuvent être utilisées pour aider l'apprenant à atteindre des buts proposés par des vues "virtuelles".

\subsubsection{Considérer le modèle usager comme un objet collaboratif}

A partir de ce que nous avons décrit, il est clair que le modèle usager est un élément d'information important pour l'utilisateur. Ce modèle peut servir dans d'autres environnements d'apprentissage pour les personnaliser mais aussi comme des données pour la recherche d'experts (nous nous approchons ici du domaine de la capitalisation de connaissance). L'utilisateur peut voir le modèle usager du LG comme une sorte de passeport décrivant ses compétences et comportements. S'il accepte de partager ce passeport avec quelqu'un d'autre, cet objet peut être utilisé comme un véritable outil collaboratif. Dans une session pédagogique, l'enseignant et l'étudiant peuvent avoir tous les deux une idée sur la manière dont ce dernier peut améliorer ses compétences personnelles ou son comportement. Ces vues ne sont pas nécessairement les mêmes. Un étudiant peut vouloir se concentrer sur une amélioration comme "être encore meilleur dans la programmation informatique" tandis que l'enseignant trouvera plus important pour cet étudiant d'améliorer ses compétences en langue étrangère" ou encore d'adopter un comportement plus collaboratif". Toutes ces considérations nous ont menés à étendre notre modèle usager pour proposer ces différentes vues. 
En partant du modèle usager actuel (vue réelle) pour un étudiant particulier (calculé comme défini précédemment), l'étudiant voudrait améliorer certaines caractéristiques de son avatar (exactement comme un utilisateur essaye de faire évoluer son avatar dans un RPG, ce qui est un point de motivation fondamental dans de tels jeux). En définissant ces améliorations souhaitées, il définit son objectif (ou cible) personnel, une vue de modèle usager virtuelle future. En parallèle, l'enseignant peut avoir sa propre vision des améliorations nécessaires et lui-même définir un objectif académique, une autre vue de modèle usager virtuelle future. Cet objet peut ainsi prendre plusieurs formes et peut être un objet de négociation pour faire un plan d'action pour l'amélioration d'un étudiant particulier. Cette approche se révèle plus riche qu'un simple relevé de notes parce que les aspects comportementaux sont présents également.

Nous décrivons maintenant comment ces actions sont possibles sur le modèle usager.

\section{Cible ou objectif personnel}

Cette vue est interactive tandis que celle réelle est statique. Pour définir des buts personnels (des niveaux à atteindre), l'apprenant peut agir sur des curseurs, accorder une valeur cible à certaines caractéristiques (voir figure 6). Une telle vue aidera l'apprenant à garder à l'esprit ses vœux et quels exercices d'apprentissage favoriser pour atteindre ces objectifs. 


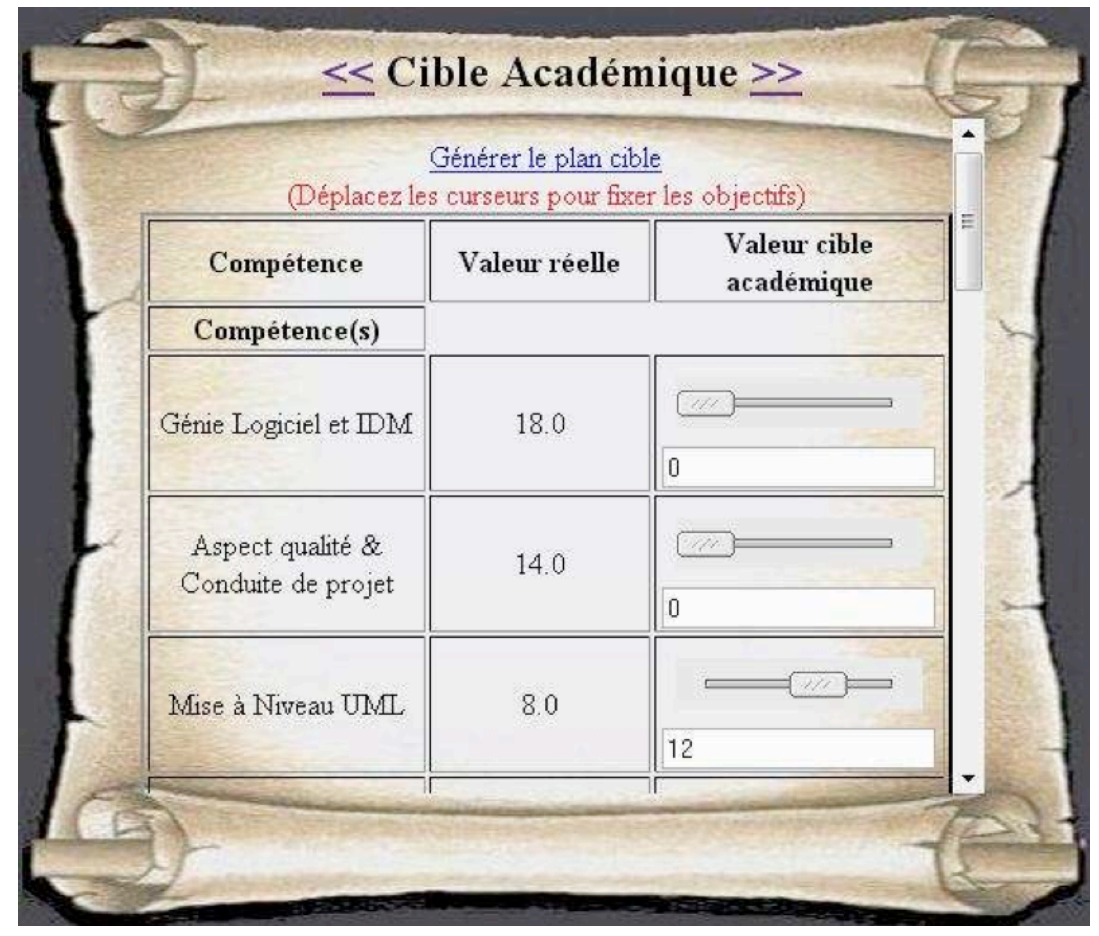

Figure 6. Exemple de “Cible Personnelle" : vue sur le modèle usager pour Learning Games

\section{Cible ou objectif académique}

Cette autre vue interactive est consacrée aux apprenants, mais seulement modifié par les enseignants. Quelques curseurs peuvent être "poussés" pour conseiller à l'étudiant de faire un effort spécifique sur une caractéristique particulière (la compétence, le comportement ou la préférence) : "atteignez cette valeur dans un tel domaine, soyez plus coopératif, soyez moins bavard, utilisez plus le chat ou cet outil, améliorez votre anglais, etc." (Voir la vue résultante pour l'étudiant dans la figure 7). 


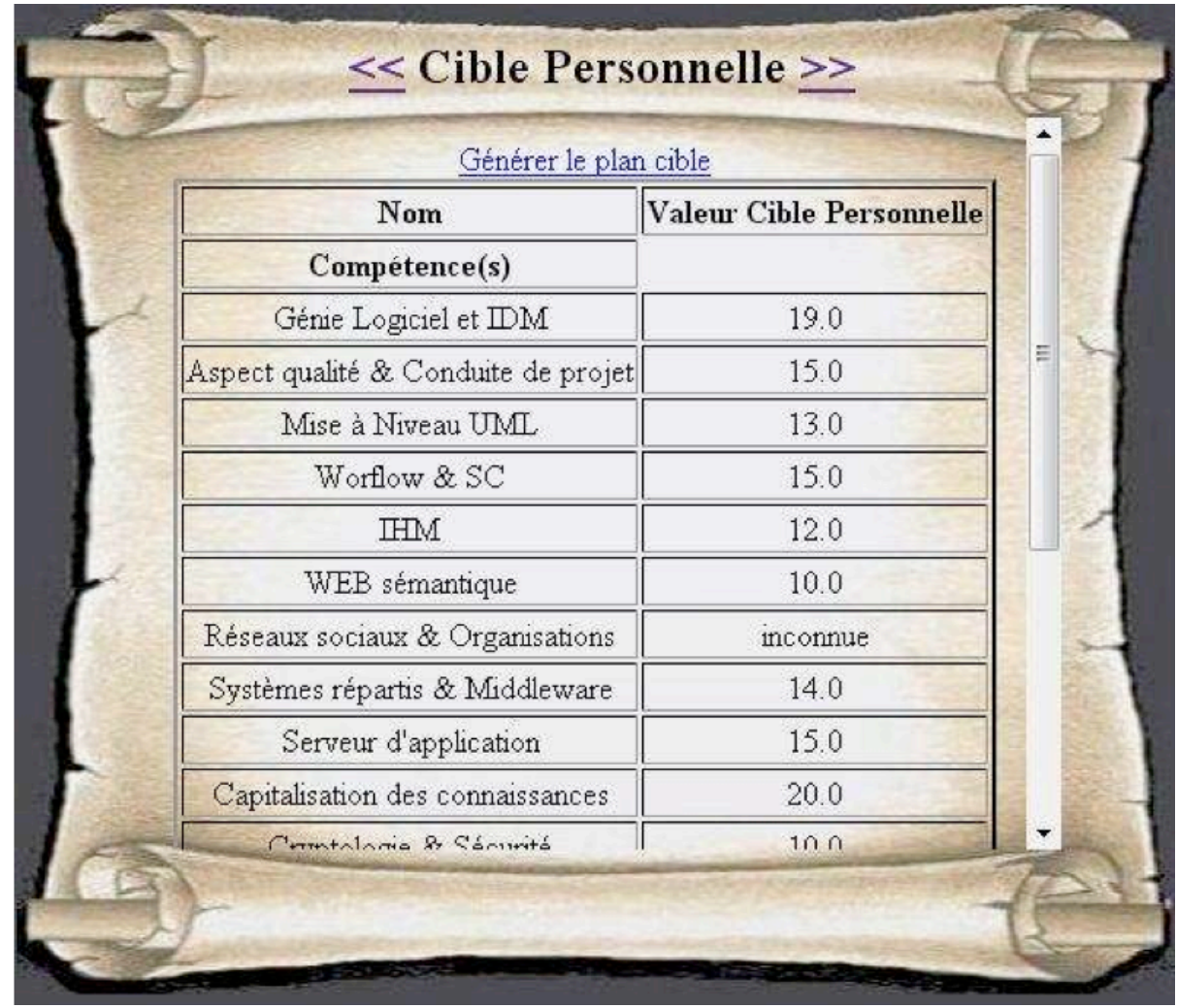

Figure 7. Exemple ${ }^{5}$ de "Cible Académique" : vue du modèle usager pour Learning Games

En outre, le système peut fournir un plan d'action pour atteindre les objectifs décrits dans les vues cibles en utilisant les règles et les valeurs des différentes caractéristiques (calculé comme décrit dans la figue 4).

\section{Génération de plan pour atteindre les objectifs définis dans les vues virtuelles}

Les métadonnées sont associés à chaque action pédagogique (scénario, exercice, quizz ou n'importe quelle action d'apprentissage semblable) disponible dans l'environnement. Ces métadonnées décrivent quelles caractéristiques, l'action pédagogique est sensée améliorer et la portée d'application de l'exercice. Ce dernier point nous permet de prendre en compte le niveau de difficulté d'un exercice. S'il est simple et si l'utilisateur est déjà un expert, il ne gagnera pas d'expérience en le

${ }^{5} \mathrm{NB}$ : Ici, non interactive car connecté comme propriétaire mais pas comme enseignant. 
faisant. Il en est de même si un exercice est difficile et l'utilisateur est un novice dans le domaine concerné.

Pour chaque objectif choisi dans une vue virtuelle, il est possible d'ajouter un plan d'activités à réaliser pour l'atteindre. Par exemple, un plan d'activité peut proposer de nouveaux scénarios choisis pour améliorer une valeur particulière concernant une compétence ou un comportement qui doit être amélioré. Comme indiqué partiellement dans la figure 8 , une activité est composée d'une description (son nom), un but et un ensemble d'actions qui permettent à ce but d'être atteint. Le plan d'action est produit à partir de l'ensemble des caractéristiques qui doivent être améliorées et de la description des actions pédagogiques disponibles. Les exercices applicables qui permettent aux caractéristiques identifiées d'être améliorés sont ajoutés au plan.

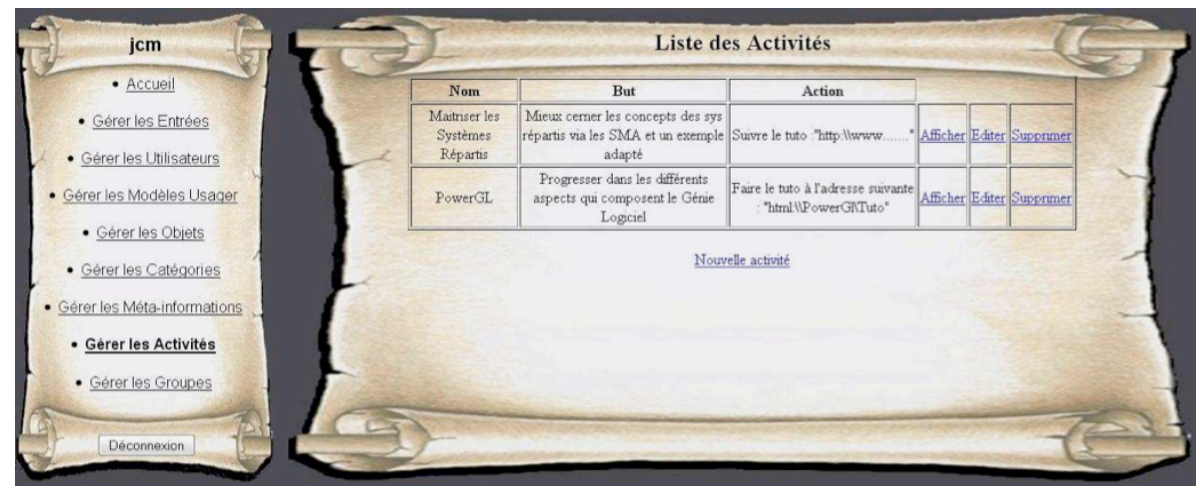

Figure 8. Plan d'activité

Toutes les propositions que nous avons faites sur la définition du modèle usager dans les LG s'appliquent aux utilisateurs individuels. Cependant, comme déjà mentionné dans l'introduction, les LG sont bien adapté à soutenir la collaboration dans le processus d'apprentissage. Nous proposons maintenant d'étendre le concept de modèle d'usager aux groupes d'apprenants.

\section{Modèle usager de groupe}


Pour l'enseignant et dans le contexte d'apprentissage collaboratif, nous offrons la possibilité de gérer les caractéristiques de groupes d'étudiants. L'administrateur du groupe est généralement l'enseignant et pour chaque caractéristique choisie, il doit connaître le minimum, la moyenne et les valeurs maximales. Par exemple, définir un modèle de groupe spécifique est bien adapté pour évaluer un groupe de projet dans une pédagogie à base de projet (voir la figure 9).

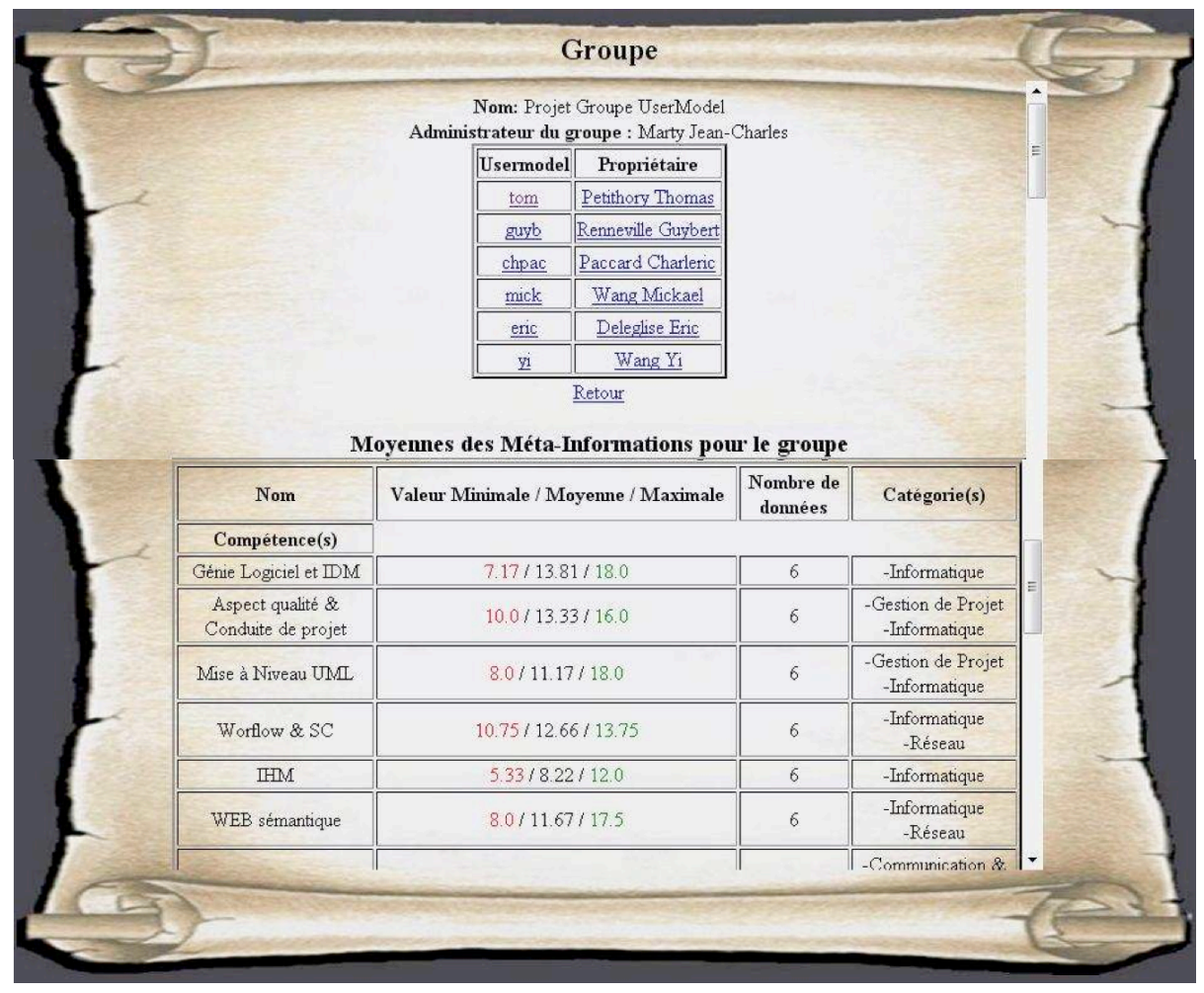

Figure 9 Exemple de modèle usager de groupe

Tous les constats présentés pour des individus concernant leurs modèles usager restent vrais pour des groupes. Il est donc possible pour un enseignant de mettre quelques objectifs au moyen du modèle usager de groupe. Cependant, la génération de plans peut être quelque peu délicate parce que les actions peuvent être appropriées à quelques personnes et pas à d'autres. Cela signifie que de nouvelles 
métadonnées sur des actions pédagogiques concernant l'amélioration de groupe doivent être définies.

Comme dit précédemment, les RPG se concentrent sur le joueur et son évolution dans le jeu. De même, habitué à pratiquer les jeux vidéo, il est naturel pour l'apprenant de faire évoluer son avatar. Néanmoins, même dans des LG récents, notre expérience montre qu'un point particulier est rarement pris en compte : le «game design» entier n'est pas relié avec les concepts d'apprentissage. Par conséquent, les étudiants ne se sentent pas immergés dans un monde qui est cohérent pour eux. Suivre les améliorations au travers du modèle usager constitue un facteur essentiel d'immersion, mais comme nous verrons dans la section suivante, l'immersion doit être envisagée d'une façon plus large et plus profonde.

\section{Quelques directives pour renforcer l'immersion dans les LG}

Dans cette partie, nous expliquons comment soutenir une immersion profonde dans le jeu dans le but d'augmenter la focalisation sur les tâches pédagogiques aussi bien que sur la collaboration entre les acteurs. L'immersion, point clé pour la motivation et la collaboration dans le jeu, est consacrée à plusieurs concepts qui peuvent être trouvés dans beaucoup d'études concernant des jeux vidéo (Woyatch, 2008)(Crawford, 1984)(Laurie et al., 2003). Il y a aussi un autre aspect que l'on doit considérer : quand les étudiants apprennent quelque chose dans un jeu, ils doivent transférer la connaissance acquise à la réalité. Si le jeu inclut les éléments qui correspondent à une situation réelle, ce transfert sera simplifié. Avec cette vue, de la même façon que (Gee, 2007) concernant les jeux vidéo et l'apprentissage, nous voudrions donner quelques directives (indiquées par $D i$ en l'italique dans la suite du texte) pour prendre en compte autant que possible la caractéristique immersion en concevant le jeu éducatif. Nous avons voulu nous concentrer sur 3 concepts qui peuvent être généralement trouvés dans la théorie des jeux vidéo classique : Game Design $^{6}$, Game Play et métaphores cohérente.

\subsection{Game design}

Un RPG pour apprendre propose d'habitude de transférer l'étudiant dans un monde virtuel. Tout d'abord, il n'est pas toujours possible pour les designers de jeu (temps, coût) de développer un monde en accord avec les dernières technologies et

\footnotetext{
${ }^{6}$ Une traduction française de ces termes ne se révèle pas satisfaisante car les termes français connotés nous semblent plus restrictifs.
} 
graphiquement très réaliste. Néanmoins, il est important de proposer un environnement cohérent et consistant. De notre point de vue, un environnement bien connu, semblable à des jeux célèbres ou des styles de jeux bien connus (Zelda ou le monde de Mario, Final Fantasy) aidera les étudiants à s'immerger dans le jeu car ils ont déjà l'impression de le connaître. Beaucoup de références, indices ou notes d'humour additionnelles ajoutées dans le design du jeu sont utiles pour renforcer cet aspect. L'idée est de provoquer le désir d'aller plus loin, explorer le monde et trouver d'autres références drôles ou agréables. Comme indiqué par le succès de la console de Wii (le Nintendo), la qualité de graphisme n'est pas un point clé.

D1: Développez un monde cohérent. Pour des raisons de cô̂t, il peut être utile de réutiliser l'apparence et les sensations de jeux bien connus...

Le game design concerne aussi la carte du jeu. Pour faire des liaisons entre le réel et les mondes virtuels, cette carte devrait correspondre autant que possible à des locaux réels où les sessions d'apprentissage ont d'habitude lieu (salles de classe, bâtiments).

D2: Trouvez les endroits réels ou les personnes d'habitude impliqués dans le processus d'apprentissage que vous voudriez intégrer dans le jeu. Pensez "aux petites histoires drôles" qui font partie du contexte d'apprentissage.

Concernant le domaine éducatif, il est encore plus important de se concentrer sur le gameplay et "casser/briser les règles du jeu".

\subsection{Game play}

Le gameplay peut être compris comme l'ensemble des règles du jeu (Djaouti et al., 2008). Ici, l'idée est de créer un effet surprenant en changeant les règles tant avec le domaine éducatif que le jeu. Par exemple, quand nous avons d'abord expérimenté notre étude du jeu, nos étudiants ont été vraiment étonnés d'être ensemble dans le même monde de jeu, de pouvoir voir les actions de leurs camarades et d'être encouragés à échanger des idées dans le jeu au moyen d'un outil de messagerie instantanée (Carron et al., 2007). Toutes les règles du jeu peuvent être tirées d'un scénario général, une histoire cohérente représentant le but pédagogique général.

D3: Concevez un scénario pédagogique complet, une histoire, ayant lieu dans les locaux définis dans le game design. 
De nouveau, nous devons montrer explicitement que ce jeu est adapté ou conçu pour les acteurs particuliers qui apprendront dans cet environnement. Les quêtes, les objets conçus / $\mathrm{PNJ}^{7}$ devraient avoir une référence explicite à ceux qui leur correspondent dans le monde réel. Par exemple, la cafétéria représentée dans le jeu est fermée pendant quelque temps parce que les étudiants n'ont pas respecté le travail des agents de nettoyage! (Une règle réelle). Comme nous verrons dans la section suivante, un des exemples les plus réussis est d'amener l'enseignant directement à l'intérieur du jeu et donner la possibilité aux étudiants d'interagir (pour demander un peu d'aide) directement avec lui par la messagerie instantanée. D'autres enseignants (non directement impliqués dans le processus d'apprentissage) peuvent aussi être ajoutés comme PNJ.

D4: Trouvez quelles règles du monde réel, vous devez reproduire dans le jeu. Certaines peuvent être là pour améliorer la crédibilité du monde virtuel, d'autres sont directement liées au processus d'apprentissage (degré de collaboration permis, par exemple).

\subsection{Métaphores cohérentes}

Le dernier point concerne la cohérence du monde du jeu lui-même. Généralement, un monde spécifique, souvent onirique, est conçu et utilisé comme support pour toutes les activités d'apprentissage. Le résultat est que le jeu est rempli avec un contenu pédagogique non adapté à l'environnement du jeu lui-même. Nous voulons souligner le fait que le game design et le game play doivent être liés avec les concepts d'apprentissage pour créer un jeu plus cohérent et garder l'apprenant immergé dans l'activité.

Les métaphores sont une façon puissante de garder une cohérence forte dans l'environnement, parce que les différents apprenants peuvent les comprendre facilement. Par exemple, le LMS développé à l'Université de Savoie a été appelée le cartable électronique (Chabert et al., 2005); nous avons proposé "un casier» électronique pour stocker "de grands" documents, le "satellite» pour observer ce qui se passe dans le LMS et cela a aidé beaucoup à une prise en main facile de l'environnement pour les nouveaux utilisateurs. Nous croyons que la même sorte de métaphores doit être insérée dans le jeu pour lier le contenu d'apprentissage avec l'environnement du jeu. Par exemple, si vous voulez enseigner une langue étrangère, les joueurs découvriront un nouveau pays (une partie spécifique de la carte de jeu) où tous les $\mathrm{PNJ}$ correspondants communiqueront au moyen de la langue étrangère. Quelques indices symbolisant le pays peuvent être ajoutés au monde pour renforcer le sentiment de cohérence.

\footnotetext{
${ }^{7}$ PNJ : Personnage Non Joueur qui généralement propose des quêtes aux joueurs.
} 
De plus, concernant le gameplay général, une réponse correcte à un exercice peut donner la clé/l'accès à un autre endroit. C'est plus simple que de devoir expliquer que "la connaissance à acquérir dans cette activité est un pré-requis pour le concept suivant à apprendre dans la session pédagogique". Le joueur n'est pas donc déconnecté du monde du jeu en passant du jeu à une caractéristique d'apprentissage spécifique. Cela nous permet de garder l'apprenant immergé dans le jeu.

D5: Définissez des métaphores liées à l'histoire générale (D3) et avec les différents objets d'apprentissage.

Dans la partie suivante, nous expliquons comment nous avons mis en œuvre ces caractéristiques dans un environnement d'apprentissage à base de jeu et nous décrivons comment nous avons conduit une expérience avec cet environnement.

\section{Learning Adventure et expérimentations}

Description de Learning Adventure (L.A.)

Dans cette partie, nous décrivons d'abord un environnement d'apprentissage à base de jeu que nous avons développé. Cet environnement servira d'un exemple pour illustrer les idées décrites dans des parties 2 et 3 . Nous expliquons les relations entre une session d'apprentissage et les objets dans le jeu et nous décrivons la mise en œuvre d'une session d'apprentissage avec des étudiants. Nous exposons alors comment la collaboration a lieu dans L.A. Nous fournissons à l'utilisateur des conseils sur la façon d'utiliser le modèle usager dans ce jeu pour adapter le scénario utilisé par les apprenants. Nous renforçons la fonction du modèle usager en le considérant aussi comme un objet interactif, sur lequel nous pouvons exécuter des actions, comme l'interaction ou la visualisation. L'interaction avec le modèle usager ouvre de nouvelles perspectives dans lesquelles l'utilisateur peut agir sur sa propre représentation, exprimant des vœux sur les compétences qu'il voudrait améliorer.

Learning Adventure (LA) est un Game Based LMS représentant un environnement 3D dans lequel la session d'apprentissage a lieu (cf. figure10). Une carte particulière (un environnement avec des lacs, des montagnes et des collines cf. directive d1-) est consacrée à un activité d'apprentissage spécifique, concernant un sujet particulier. Chaque partie de la carte représente l'endroit où une (sous)activité donnée peut être réalisée. La topologie de carte représente le scénario complet de la session d'apprentissage, c'est-à-dire le séquencement entre les activités. Il y a autant de régions que d'activités réelles et les régions sont reliées entre elles par des chemins et des gardes (PNJ), indiquant les possibilités d'accès à une activité à partir des autres. Un exemple de scénario pédagogique vu comme une topologie de carte est présenté dans la figure 10. Des modèles semblables qui lient 
des questions pédagogiques avec des éléments de jeu peuvent être trouvés avec un point de vue plus général dans (Amory et al., 1999) et plus précisément concernant cette approche dans (Carron et al., 2008).

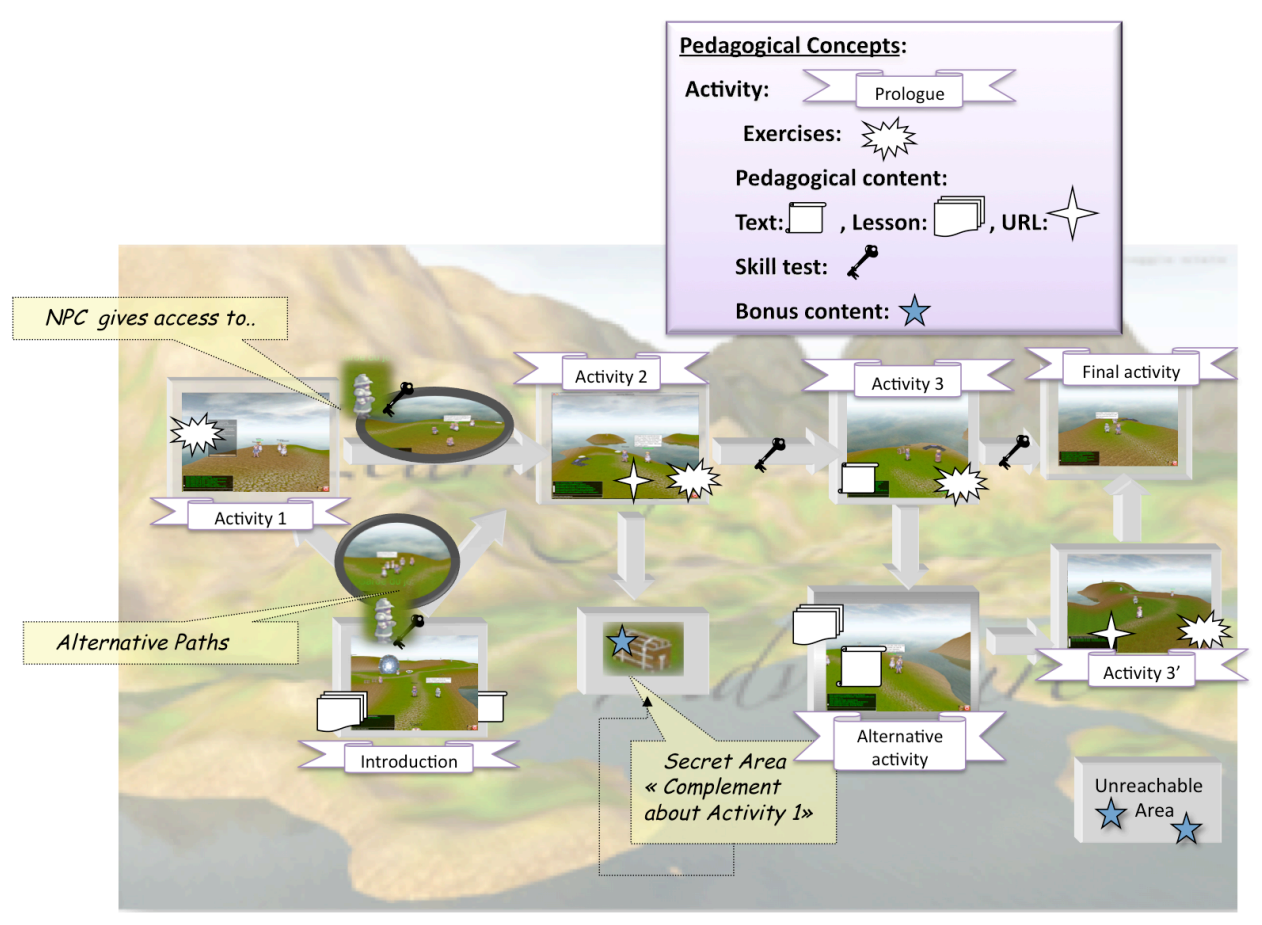

Figure 10: Un exemple de scenario pédagogique vu comme une topologie de carte de jeu.

Les joueurs (étudiants ou enseignants) éventuellement représentés par leurs propres avatars (D2), peuvent se déplacer par l'environnement, exécutant une séquence de sous-activités pour acquérir des connaissances (cf. figure 11). Les activités peuvent être effectuées d'une façon personnelle ou collaborative (voir (Dillenbourg et al., 1996) pour une liste de capacités de coopération) : vous pouvez avoir accès à la connaissance à travers des objets disponibles dans le monde, via l'aide des enseignants, ou du travail avec d'autres étudiants. Quand le besoin se fait sentir, il est facile d'avoir un résumé de la connaissance déjà acquise et du comportement général dans l'environnement. En effet, on peut facilement atteindre son modèle usager par l'icône de sac à la partie en bas à droite de l'écran. 


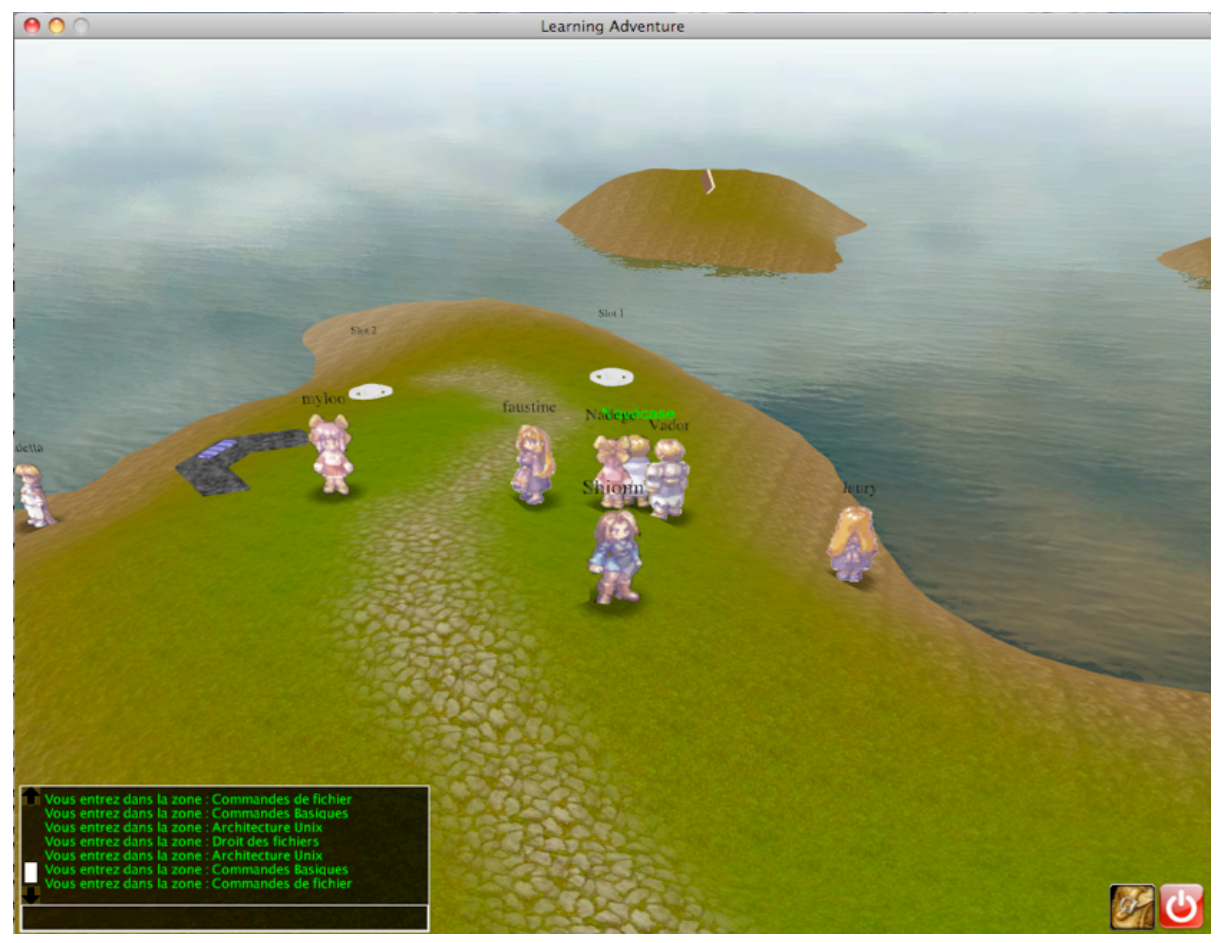

Figure 11. Copie d'écran de l'environnement Learning Adventure

De manière similaire à (Egenfeldt-Nielsen 2003), notre approche est très pragmatique et principalement basée sur des expérimentations empiriques.

\section{Une expérimentation avec L.A.}

Nous illustrons maintenant comment une session de formation peut être immersive et flexible dans un tel environnement, par une expérimentation menée à travers L.A.

- Conditions et méthodologie de l'expérimentation.

Cette expérience a été effectuée en 2009 dans notre université avec une organisation co-située: Pendant l'expérimentation, quatre groupes de quinze 
étudiants avec leur enseignant étaient présents successivement dans la salle de classe équipée de 15 ordinateurs. Concernant la perception de présence sociale (voir de Kort et al., 2008), les apprenants ont été disposés de manière à être loin les uns des l'autre, limitant le contact des yeux mutuel, les échanges naturels de l'approche (indices, imitation). Les étudiants avaient environ 18 ans et étaient familiers avec l'utilisation de l'informatique. Concernant les quatre groupes, il y avait une répartition presque égale entre garçons et filles. Chaque étudiant a eu accès à l'environnement virtuel via son poste de travail et avait une vue personnelle adaptée du monde (vue centrée sur son avatar avec possibilité de changer l'angle de vue, le zoom). Ces étudiants ont utilisé l'environnement pendant environ une heure et demi.

Il leur a été explicitement permis voire recommandé de communiquer via l'outil de messagerie instantanée fourni le système («chat» intégré à L.A.) et ils ont été avertis qu'ils seraient observés pendant l'utilisation du système. Deux observateurs étaient présents dans la salle de classe. Les étudiants étaient toutefois libres de refuser cette observation (le même travail pratique était disponible à l'extérieur de l'environnement apprenant), mais chacun a consenti à suivre le protocole proposé. Finalement, absolument aucune indication n'a dû être donnée concernant l'utilisation du jeu (ce qui est un avatar, comment déplacer son propre avatar, comment interagir avec les PNJ, les objets et les autres joueurs).

\section{- Objectifs pédagogiques}

Le cours servant de support à cette expérimentation était consacré au domaine des Systèmes d'exploitation. Le contenu pédagogique concernait "les commandes Unix de base". Le but de la session (RPG) était d'évaluer la connaissance et le savoir-faire des étudiants à propos d'UNIX. Une histoire guidait la quête de connaissance grâce à des métaphores (D5) : téléportant, multipliant/clonant ou détruisant des objets dans le monde représentant respectivement le déplacement, la copie et la suppression de fichiers dans le système de fichiers. Le défi est encouragé par des PNJ qui proposent un parcours cohérent, ici tester de nouvelles expériences futuristes (D3). L'immersion est renforcée quand les actions des utilisateurs ont un impact direct sur les objets du monde. Dans cette expérimentation, un artefact correspondant à la console de système était présent dans le jeu (cf. figure 12). Les actions correctement réalisées sur le système de fichiers étaient ainsi visibles dans le jeu : un livre (objet du monde) a été déplacé, dupliqué ou supprimé grâce aux actions effectives sur le livre du système de fichiers (fichier (D4)). Pour terminer, l'enseignant était présent dans le jeu avec un avatar personnel dédié (D4) : il était possible de discuter avec lui à travers le chat pour lui demander de l'aide ou des conseils par exemple. 


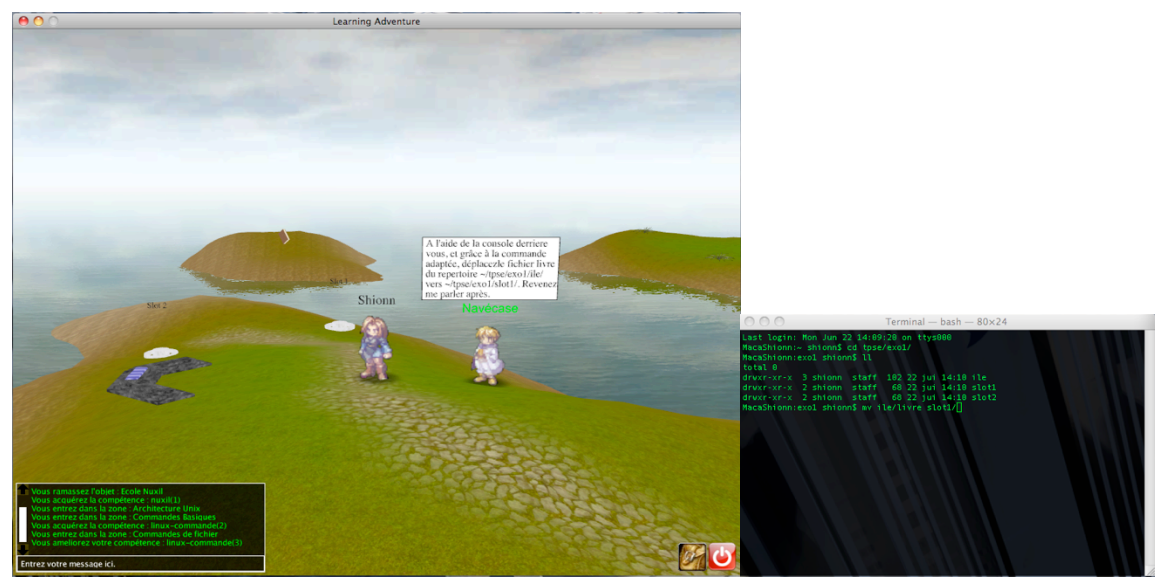

Figure 12. Téléporter un livre directement dans le jeu via une console système linux system.

- Considérations techniques:

L'environnement entier est codé en JAVA, développé avec l'aide d'un ingénieur et d'étudiants en stage. La partie de réseau s'appuie sur le projet OpenSource RedDwarf ${ }^{8}$. L'environnement entier est basé sur une architecture client-serveur. Le premier développement du modèle usager a été développé en ruby et ruby on rails (ror) pour la gestion de modèle d'usager (création et consultation) mais est maintenant en cours d'intégration à L.A. en java comme indiqué dans la figure 13.

On récompense automatiquement les actions correctes avec le niveau de compétence approprié. Le modèle usager de l'apprenant est mis à jour en conséquence dans le jeu.

Comme expliqué dans la partie 2, il est aussi possible d'agir sur le modèle usager, définir de nouvelles valeurs dans ce modèle, représentant un objectif concret à atteindre. Dans ce cas, nous pouvons attendre du système un plan généré, composé de plusieurs activités contribuant à atteindre cet objectif. Le système doit donc pouvoir détecter si un exercice existant est approprié pour l'objectif donné (cf. figure13. illustrant un tel processus).

\footnotetext{
${ }^{8} \mathrm{http}: / /$ www.reddwarfserver.org/
} 


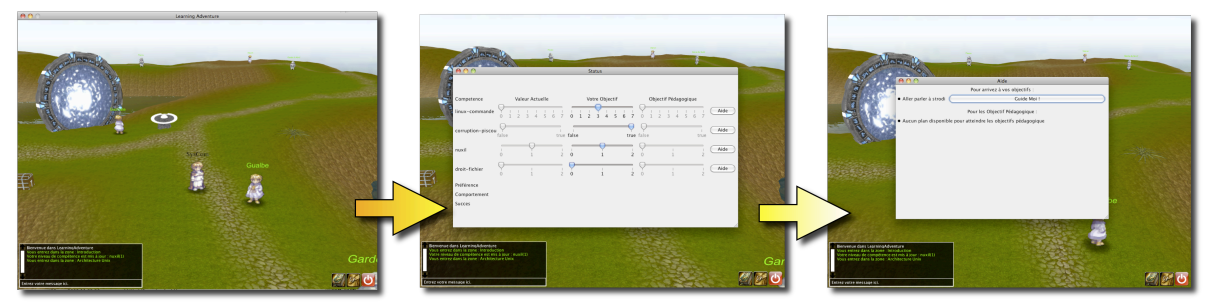

Figure 13. Programmer une progression de compétence et obtenir un plan adapté

Techniquement, les plans sont actuellement décrits dans un simple format $\mathrm{xml}$ format comme nous pouvons le voir sur la fig. 14 .

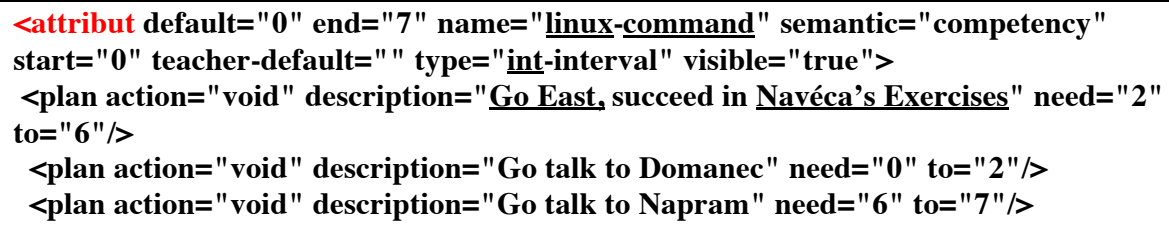

Figure 14. Exemple de plans décrit en xml.

- Evaluation et résultats

À la fin de l'expérimentation, nous avons demandé aux étudiants de remplir des questionnaires pour donner un retour sur leurs sentiments concernant leur session de travail. Douze questions (classement sur une échelle de valeur et ouvertes) ont été utilisées. Le questionnaire a évalué des aspects liés à plusieurs parties du LG (contenu pédagogique, scénario/histoire, activités collaboratives et convivialité de l'interface utilisateur).

La question finale laissait aux étudiants la possibilité de proposer quelques améliorations concernant les points faibles et forts du jeu.

L'objectif initial concernant la motivation a été atteint. Les étudiants ont préféré d'une façon unanime travailler avec cet environnement plutôt qu'un travail pratique classique sur des stations de travail et plus généralement étaient très enthousiastes au sujet de ce type d'expérience.

Dans un classement à 5 niveaux (allant de absolument pas -1- à tout à fait d'accordn-5-, les résultats étaient principalement «d'accord»-4- ou même -5concernant l'ajout d'aspects plus collaboratifs. Nous devons péciser que les activités 
choisies correspondaient très bien avec le jeu. Les résultats obtenus des questionnaires ont été confirmés par le fait que les étudiants n'ont pas fait de pause. A l'inverse de certaines expérimentations préliminaire faites sur les jeux vidéo, nos ressources pédagogiques ou des informations de fond (Egelfeldt-Nielsen, 2003) ont été bien intégrées dans le jeu et adaptées pour l'expérience de jeu. Néanmoins, quelques questionnaires suggèrent des améliorations possibles, principalement liées avec l'interface ou avec le scénario (les filles n'ont pas beaucoup aimé le scénario). A la proposition de renforcer la partie narrative (histoire servant de support aux quêtes), 33,3\% n'étaient pas d'accord, $41,7 \%$ n'avaient pas d'idée à ce sujet et $25 \%$ étaient d'accord.

Sauf concernant la perception de scénario, étudiants et étudiantes semblent réagir de la même façon à ce LG mais cela peut être en raison de la petite taille de l'échantillon qui n'est pas une vraie représentation de la population car il est maintenant bien connu que des aspects de genre spécifiques doivent être pris en considération (Hayes, 2008).

Nous pouvons remarquer que les résultats de cette évaluation sont intéressants du point de vue des développeurs de la plate-forme de jeu. Par contre, ils ne satisfont pas aux critères d'évaluation des utilisateurs qui veulent effectivement connaître si l'état de motivation est modifié en cas d'utilisation répétée de la plate-forme. Dans ce cadre, nous nous référons aux conseils méthodologiques indiqués dans (Barendregt et al., 2010) qui préconisent de se tourner vers les spécialistes du concept à évaluer (dans notre cas, la motivation traitée en sciences humaines).

La motivation est un processus complexe et, d'après (Galand, 2006), l'estime de soi est directement liée à ce processus. Le fait d'être confiant en ses capacités permet de relever des challenges plus difficiles, satisfaisant d'un point de vue intellectuel, renforçant ainsi la dynamique motivationnelle.

Une des pistes à explorer pour l'évaluation de la motivation est de mesurer l'estime de soi de l'apprenant au sein de l'environnement d'apprentissage par le jeu et de regarder son évolution. (Galand, 2006) met en avant trois indicateurs qui sont directement liés à la motivation. Le premier est le choix de l'activité d'apprentissage, la confiance en soi favorisant le choix d'activités plus complexes. Le second est la persévérance, qui peut être mesurée grâce au temps passé sur les exercices ou à la compréhension des erreurs. Le troisième concerne l'engagement cognitif qui s'identifie par les stratégies d'apprentissage choisies par l'apprenant, par son autonomie, par la prise d'initiative au niveau des parcours suivis.

Ces indicateurs devront être disponibles dans nos expérimentations futures si nous souhaitons mesurer de manière effective l'évolution de la motivation des apprenants au cours du jeu.

Il était aussi important d'interviewer l'enseignant. De son point de vue lié à une expérimentation précédente (Marty et al., 2009), il était obligatoire d'avoir des outils 
l'aidant dans sa tâche de supervision. Plusieurs indicateurs ou widgets ont été donc intégrés dans l'environnement pour satisfaire à cette exigence.

Un point important concernant la flexibilité est relatif à l'adaptation de l'observation de l'activité en cours. Les faits obtenus à partir d'observation sont appropriés seulement si ce qui est observable se réfère à un objectif. L'enseignant peut avoir besoin de changer cet objectif. Dans cette expérimentation, un widget de vue complet a été activé en début de session d'apprentissage: la progression de chaque étudiant a d'abord été contrôlée avec un haut niveau de granularité. En effet, l'enseignant a d'abord eu besoin d'une vue générale l'activité en cours. Ensuite, l'enseignant a souhaité et pu obtenir des informations plus précises sur un étudiant spécifique ayant des difficultés, grâce aux informations incluses dans le modèle usager de cet apprenant (cf. figure 15; widget inférieur : liste des étudiants connectés; widget supérieur : compétences).

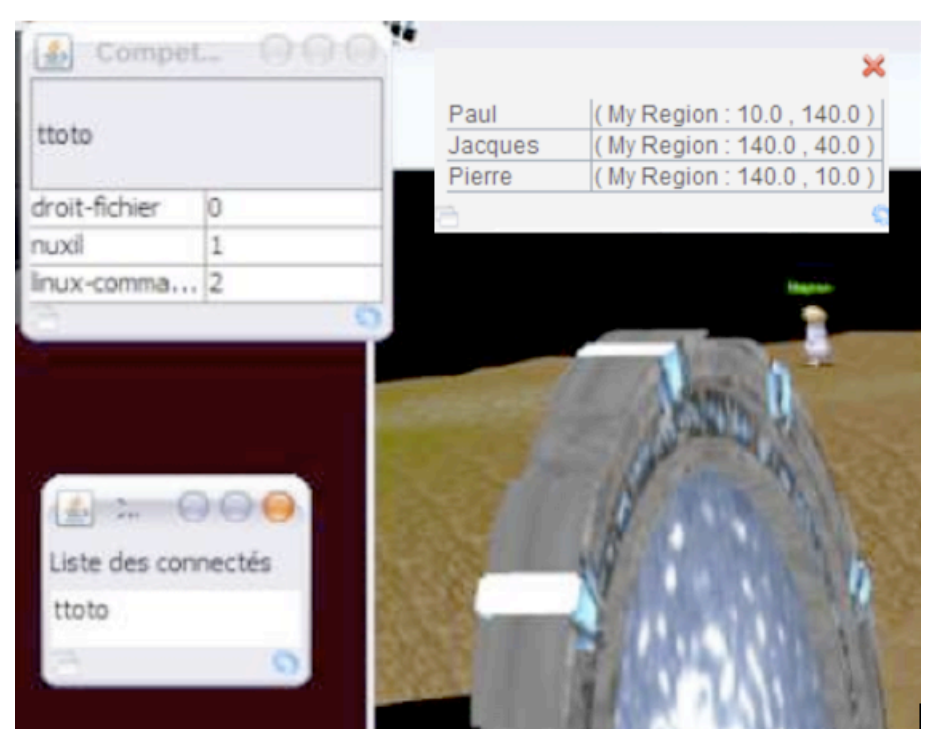

Figure 15. Indicateurs concernant un étudiant spécifique.

Nous pouvons même aller plus loin et utiliser ces indicateurs pour réguler l'activité (si les indicateurs touchent à la collaboration dans l'environnement) ou exécuter des actions de remédiation quand un apprenant a besoin d'aide. Cette caractéristique peut par exemple être appliquée à la situation liée à la figure 15 . Nous pouvons remarquer que la note associée aux droits ("droit-fichier") est très basse. Cela signifie que cet étudiant est incapable de changer les droits d'un fichier. 
Or cette compétence était particulièrement centrale dans cette expérimentation, pour par exemple, agir sur des objets nouvellement découverts. Pour achever cette quête et pour une bonne évaluation de l'apprentissage, il est important que l'enseignant puisse détecter ce problème et y réagir rapidement.

Pour améliorer l'apprentissage concernant cette compétence, le système propose trois actions de remédiation à l'enseignant : ajouter un nouveau PNJ (exercice), ajouter de nouveaux objets (ressources pédagogiques) ou envoyer un message pour obtenir une aide directe (communication entre rôles) comme indiqué dans le coin supérieur gauche de la figure 16. Ces fonctionnalités aident à renforcer l'immersion de l'enseignant dans le jeu. Cependant, cet objectif est assez ambitieux et nécessiterait une classification complète des actions en termes de remédiation. Cette tâche importante peut actuellement seulement être faite pour un monde restreint.

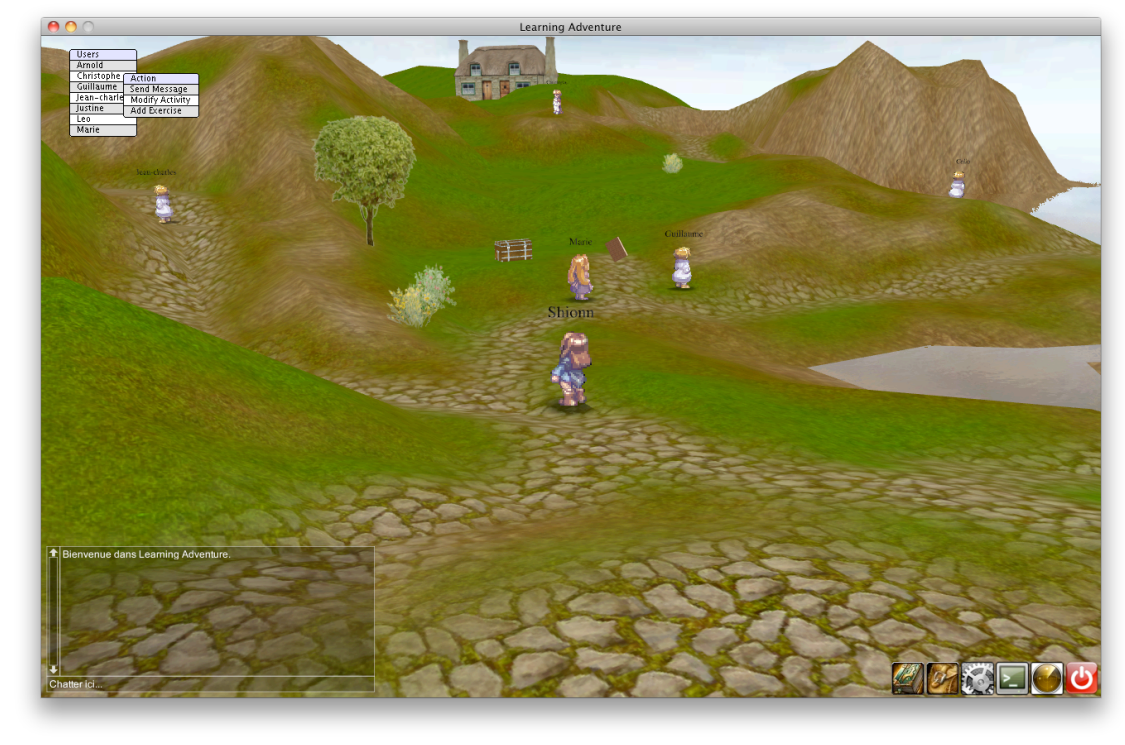

Figure 16. Indicateurs et réactions possibles concernant un étudiant particulier

Nous pouvons remarquer que de tels environnements sont très génériques et permettent de jouer à tout moment, de partout et sur des sujets d'étude différents. Plusieurs étudiants nous ont demandé de pouvoir compléter ou refaire une quête plus tard. Il est donc important d'inclure des fonctions persistantes pour construire une connaissance composée tout au long du temps. Le modèle usager est un concept clé permettant de déclarer les compétences et les comportements d'un apprenant particulier. Dans cet objectif, nous sommes actuellement en train d'adapter Learning Adventure pour avoir un monde persistant. 


\section{Conclusion}

Dans cet article, nous avons présenté notre travail autour d'un modèle usager adapté au Learning Games. Comme nous l'avons vu dans la première section, l'introduction d'un tel modèle dans un LG lève un certain nombre de questions : qu'est-ce qui est à l'intérieur d'un modèle usager adapté aux LG ? Ces informations peuvent-elles être classifiées et comment ? Comment rendre ces informations disponibles pour les utilisateurs à l'intérieur du jeu ? Est-ce graphiquement envisageable? Comment mettre à jour le modèle usager dynamiquement ? Le modèle usager peut-il servir d'objet collaboratif entre les étudiants et l'enseignant pour négocier des objectifs pédagogiques ? Nous avons répondu à toutes ces questions dans cette partie en soulignant le besoin d'un modèle d'usager spécifique et flexible adapté aux Learning Games.

Nous avons même proposé un modèle de groupe, consolidant notre croyance que la génération suivante d'environnements d'apprentissage à base de jeu prendra en compte la dimension sociale. Mais comme indiqué dans la deuxième partie, l'immersion est un autre concept clé qui doit être pris en considération en concevant un LG. Nous avons ainsi proposé quelques directives pour respecter cette autre exigence. En outre, il est important de mettre e, place des indicateurs visuels de collaboration pour avoir une meilleure conscience de ce qu'il se passe lors d'une session d'apprentissage collaborative afin d'être en mesure de réguler les interactions sociales entre les acteurs.

\section{BibLIOGRAPHIE}

Amory, A., Naicker, K., Vincent J. and Adams, C. (1999). The use of computer games as an educational tool: Identification of Appropriate Game Types and Game Elements. British Journal of Educational Technology 30(4), 311-321.

Barendregt W. and von Feilitzen M. (2010), Attacking Immune Attack ${ }^{T M}$ ? An Evaluation by Teacher Students, $4^{\text {th }}$ European Conference on Games Based Learning. Copenhaguen, Denmark, 2022 October.

Bogost, I. (2007). Persuasive Games: The Expressive Power of Videogames. Cambridge: MIT Press.

Brusilovsky, P. (2001). Adaptive hypermedia. User Modeling and User Adapted Interaction, Ten Year Anniversary Issue (Alfred Kobsa, ed.) 11 (1/2), 87-110.

Buckingham, D. and Burn, A. (2007). Game Literacy in Theory and Practice. Journal of Educational Multimedia and Hypermedia 16(3), 323-349.

Carron, T., Marty J.C., Heraud J.M., France L. (2006). Helping the teacher to re-organize tasks in a collaborative learning activity: an agent based approach, Sixth IEEE International Conference on Advanced Learning, ICALT'06, pp 552-554, Kerkrade, Netherlands.

Carron T., Marty J.C., Heraud J.M., France L. (2007). Games as Learning Scenarios: are you Serious?, European Conference on Games Based Learning (Scotland). 
Carron, T., Marty J.-C., Heraud J.-M. (2008). Teaching with Game Based Learning Management Systems: Exploring and observing a pedagogical dungeon, Simulation \& Gaming Special issue on eGames and Adaptive eLearning.

Chabert G., Marty J.-C., Caron B., Carron T., Vignollet L., Ferraris C. (2005). The Electronic Schoolbag: a CSCW workspace, AI \& Society: The Journal of Human-Centred Systems and Machine Intelligence.

Choukroun J., Lieury A. (1985): Rôle du mode de présentation (visuel, auditif, audio-visuel) dans la mémorisation d'instructions, L'année psychologique, 1985, n 4, pp. 503-516.Consulted april $24^{\text {th }} 2009$. DOI: $10.3406 /$ psy. 29110. url : http:/ / www.persee.fr/web/revues/home/prescript/article/psy_000350331985 num 85429110

Crawford, C. (1984). The Art of Computer Game Design", Eds. Osborne/McGraw-Hill.

De Kort Y.A. W. and Ijsselsteijn W.A. (2008). People, Places, and Play: Player Experience in aSociospatial Context. Computers in Entertainment 6(2).

Dillenbourg P., Baker M., Blaye A., O'Malley C. (1996). The evolution of research on collaborative learning. Learning in Humans and Machine: Towards an interdisciplinary learning science, pp. 189-211.

Dimitracopoulou, A., Bollen, L., Dimitriadis, Y., Harrer, A., Jermann, P., Kollias, V., Marcos, J., Martinez, A., Pedrou, A. (2005). State of the art of interaction analysis for Metacognitive support $\mathcal{E}$ diagnosis, in IAJEIRP.

Dimitriadis, Y., Antonio Marcos, J., Martínez, A., Anguita, R. (2006). Interaction Analysis for the Detection and Support of Participatory Roles in CSCL, CRIWG, pp155-162.

Djaouti D., Alvarez J., Jessel J-P, Methel G., Molinier P. (2008). A Gameplay Definition through Videogame Classification, International Journal of Computer Game Technology, Hindawi Publishing Corporation.

Dondlinger, M.J. (2007). Educational Video Game Design: A Review of the Literature. Journal of Applied Educational Technology 4(1), 21-31.

Egenfeldt-Nielsen, S. (2004). Practical Barriers in Using Educational Computer Games. On the Horizon 12(1), 18-21.

Ermi, L. and Mäyrä, F. (2005). Fundamental Components of the Gameplay Experience: Analysing Immersion. In print proceedings, Changing Views: Worlds in Play, Digital Games Research Association Conference. Tampere: University of Tampere Press.

Fink, J. and Kobsa, A. (2000): A review and analysis of commercial user modeling servers for personalization on the World Wide Web. User Modeling and User-Adapted Interaction 10(23), Special Issue on Deployed User Modeling, pp209-249.

Galand, B. (2006) Réussite scolaire et estime de soi. L'école en question. Revue Sciences Humaines, Hors série spécial n ${ }^{\circ} 5$.

Galarneau, L. and Zibit, M. (2007). Online Game for $21^{\text {st }}$ Century Skills. In Games and Simulations in Online Learning: Research and Development Frameworks, edited by D. Gibson, C. Aldrich and M. Prensky (pp.59-88). Hersey: Information Science Publishing.

Gee, J.P. (2007). Good Video Game + Good Learning: Collected essay on Video Games, Learning, and Literacy. New York: Palgrave MacMillan.

Gendron E., Carron T., Marty J.-C., (2008). Collaborative indicators in Learning Games: an immersive factor., $2^{\text {nd }}$ European Conference on Games Based Learning. Barcelona, Spain, 16-17 October.

Hayes, E. (2008). Girls, Gaming and trajectories of IT Experience. In Beyond Barbie and Mortal Kombat: New Perspectives on Gender and Gaming, edited by Y. Kafai, C. Heeter, J. Denner and J. Sun (pp.217-230). Cambridge: MIT Press.

Hijon R., Carlos R., (2006) E-learning platforms analysis and development of students tracking functionality, in Proceedings of the 18th World Conference on Educational Multimedia,Hypermedia \& Telecomunications, pp. 2823-2828.

Kahne, J., Middaugh, E. and Evans, C. (2009). The civic Potential of Video Games. Cambridge: MIT Press.

Kian-Sam H., Chee-Kiat K. (2002) Computer Anxiety and Attitudes toward Computers among Rural Secondary School Teachers: A Malaysian Perspective, Journal of Research on Technology in Education, Vol. 35 Issue 1, pp. 27-49.

Kirriemuir, J. and A. MacFarland (2004). Literature Review in Games and Learning. FutureLab. http:/ / hal.archives-ouvertes.fr/docs/00/19/04/53/PDF/kirriemuir-j-2004-r8.pdf (accessed 6 April 2010) 
Kobsa, A. (2001). Generic User Modeling Systems, in User Modeling and User-Adapted Interaction 11(1-2), pp 49-63.

Laurie N. Taylor. (2003), Video Games: Perspective, Point-of-View, and Immersion, Thesis. http://www. gamasutra.com/education/theses/20030818/taylor_01.shtml\#.

Loghin G., Marty J.-C., Carron T. (2008). A flexible agent-based observation solution for educational platforms, in IEEE International Conference on Advanced Learning, ICALT'08.

Marty J.-C., Heraud J.-M., France L., Carron T. (2007) Matching the Performed Activity on an Educational Platform with a Recommended Pedagogical Scenario: a Multi Source Approach, Journal of Interactive Learning Research, special issue on Usage Analysis of Learning Systems: Existing Approaches and Scientific Issues. Vol 18, N², 27p.

Marty J.-C., Carron T., Heraud J.-M. (2009). Observation as a requisite for games based learning environments, chapter IV of the book: Games Based Learning Advancements for multisensory human computer interfaces: technics and effective practices - May 2009. pp. 51-71.

Matheas, M. (2005). Procedural Literacy: Educating the New Media Practitioner. On the Horizon 13(2), 101-111.

Prensky, M. (2000). Digital Game-Based Learning. New York: MacGraw Hill.

Rueda U., Larranaga M., Arruarte A. and Elorriaga J.A. Dynamic Visualization of Student Models using Concept Maps. Proceedings of the 11th International Conference on Artificial Intelligence in Education, p 89-96, 2003.

Salen, K. (2007). Gaming Literacies: A Game design study in action. Journal of Educational Multimedia and Hypermedia 16(3), 301-322.

Squire, K. (2003). Videogames in Education. International Journal of Intelligent Games \& Simulations 2(1), 49-62.

Vassileva J., McCalla G., Greer J. (2003). Multi-Agent Multi-User Modeling, User Modeling and User-Adapted Interaction, 13:(1), 179-210. Special Issue on User Modeling and Intelligent Agents

Vygotski L.S. (1934), Language and Thought, Gosizdat, Moscow.

Woyach S. (2008), "Immersion Through Video Games", in illumin review of engineering in everyday Life, issue iv, vol.5 url: http://illumin.usc.edu/article.php?articleID=103 (Accessed 4 April, 2009).

Yu T. W. (2009). Learning in the Virtual World: the Pedagogical Potentials of Massively Multiplayer Online Role Playing Games. In International Education Studies. Vol. 2(1). 